\title{
Influência da exposição à fumaça de cigarro e do treinamento físico no apetite e desenvolvimento corporal em ratos
}

Influence of exposure to cigarette smoke and physical training in appetite and body development in rats

Carmen Castardeli*

\section{Resumo}

Elisvaldo Alves Ramos**

Paula Grippa Sant'Ana**

André Soares Leopoldo**

Ana Paula Lima Leopoldo**

Edson Castardeli**

O fumo apresenta potencial para promover alterações no sistema hormonal, apetite e, consequentemente, na ingestão alimentar. Por sua vez, o treinamento físico amplia a demanda metabólica, exerce efeito substancial sobre o balanço energético. Objetivou-se verificar a influência do tabagismo e do treinamento físico no apetite e no desenvolvimento do peso corporal em ratos. Utilizou-se ratos Wistar ( $n=40$, entre $200 \mathrm{~g}$ e $250 \mathrm{~g}$ ) distribuídos em quatro grupos: Controle (C); Controle Treinado (CT); Exposto (E); Treinado/Exposto (TE). A exposição à fumaça de cigarro (EFC) e treinamento (natação) durou 15 semanas. Os animais foram pesados semanalmente e a ingestão alimentar pesada diariamente. Analisou-se com ANOVA de duas vias e adotou-se nível de significância de 5\%. A ingestão nos ratos EFC foi menor, comparado com os não $\mathrm{EFC}(\mathrm{p}=0,001)$. Os ratos treinados apresentaram menores valores na ingestão em relação aos não treinados $(p=0,044)$. A interação estatística entre os fatores (fumo e natação) interferiu na ingestão $(p=0,013)$. As curvas de crescimento apresentaram valores inferiores de peso corporal nos grupos EFC. Houve interação estatística entre os fatores fumo e natação para o peso corporal $(p=0,046)$. Ocorreu diferença estatística com maiores valores para os pesos do coração, pulmão e tíbia nos grupos não EFC, em comparação aos ratos EFC. Conclui-se que a EFC inibe o apetite e promove prejuízo no desenvolvimento corporal. E, o treinamento físico apresenta influência no balanço energético negativo cronicamente, com diminuição no ganho de peso. Porém, não interfere no desenvolvimento corporal, sendo benéfico diferentemente da exposição à fumaça de cigarro.

Palavras-chave: Fumo. Tabagismo. Ingestão alimentar. Atividade física. Apetite.

\begin{abstract}
The tobacco has the potential to cause changes in the hormonal system, appetite and therefore food intake. In turn, physical training increases metabolic demand, exerts a substantial effect on the energy balance. This study aimed to verify the influence of smoking and physical training in appetite and the development of body weight in rats. Rats Wistar were used ( $n=40$, between $200 \mathrm{~g}$ and 250g) rats were distributed into four groups: control (C); Trained control (TC); Exposure (E); Trained / exposure (TE). Exposure to cigarette smoke (ECS) and training (swimming) lasted 15 weeks. The animals were weighed weekly and food intake weighed daily. Analyzed with two-way ANOVA and was adopted a significance level of $5 \%$. The ECS intake in rats was lower compared to the non ECS ( $p=0.001)$. The trained rats had lower values in intake compared to untrained $(\mathrm{p}=0.044)$. The statistical interaction between factors (smoking and swimming) interfered with the intake ( $p=0.013$ ). The growth curves showed lower body weight in the groups ECS. Was no statistical interaction between smoking and factors swimming to body weight $(p=0.046)$. Statistical difference with higher values for the weights of the heart, lung, and tibia in group no ECS, compared to rats ECS. We conclude that ECS inhibits appetite and promotes loss in body development. And, physical training has chronically negative influence on energy balance, with reduction in weight gain. However, it does not interfere with body development, and beneficial unlike exposure to cigarette smoke.
\end{abstract}

Keywords: Smoke. Smoking. Dietary intake. Physical activity. Appetite.

DOI: 10.15343/0104-7809.20164002169179

\footnotetext{
* Universidade Federal do Espírito Santo - UFES, Departamento de Ciências Fisiológicas, Vitória, Espírito Santo/ES, Brasil.

** Universidade Federal do Espírito Santo - UFES, Departamento de Desportos, Vitória, Espírito Santo/ES, Brasil.

Os autores declaram não haver conflitos de interesse.
} 


\section{INTRODUÇÃO}

O fumo é composto por cerca de 6.700 substâncias, das quais 4.720 foram bem identificadas ${ }^{1,2,3}$. Essas substâncias têm duas procedências, uma é a folha do fumo e a outra é proveniente da combustão do fumo, que é a sua fumaça. A fumaça pode ser dividida em duas fases: 1) a fase do vapor, por exemplo, o monóxido de carbono, dióxido de carbono, sulfeto carbonila, benzeno entre outros gases; 2) a fase de partículas onde encontramos a nicotina, anabatina, fenol, catecol, hidroquinona e muitas outras partículas ${ }^{4,5}$.

Devido as inúmeras substâncias contidas no fumo e suas diversas propriedades, os constituintes da fumaça de cigarro têm potencial para promover alterações no sistema hormonal, mais especificamente nos hormônios que regulam a ingestão, como a leptina e a grelina $^{5}$. Esses hormônios são relacionados com a alteração no apetite e, consequentemente, controlam a ingestão alimentar e peso corporal em mamíferos ${ }^{6}$.

A leptina está ligada ao binômio apetitesaciedade e se trata de peptídico liberado pelo tecido adiposo que sinaliza ao cérebro informações sobre o estoque de gorduras, sobre o estado nutricional e modula a liberação de neuropeptídios de efeito anorexígeno e orexígeno, no organismo. Essa sinalização ao sistema nervoso central aumenta ou diminui a atividade metabólica, com consequente influência no apetite.

O uso crônico de tabaco poder aumentar a concentração sérica da leptina por dois principais mecanismos: sua maior produção e/ou a diminuição de sua depuração renal ${ }^{6,8,9}$. Os fumantes tendem a apresentarem menores índices de massa corporal, em comparação com indivíduos não fumantes. Estudos epidemiológicos transversais clássicos observaram significativa relação entre o uso regular de tabaco e o peso corporal baixo entre os fumantes quando comparados aos não fumantes ${ }^{10,11}$.

Alguns constituintes da fumaça de cigarro, como a nicotina, ativa a via dopaminérgica causa relaxamento, redução do estresse, aumento do estado de vigília, melhora da função cognitiva, modulação do humor e perda de peso ${ }^{12}$. Além disso, a nicotina é capaz de ativar centros hipotalâmicos e acelerar a atividade simpática, e consequente aumento do gasto energético, reduzindo o peso corporal, ou seja, fumantes têm a demanda calórica aumentada, devido ao seu aumento da taxa metabólica ${ }^{13}$.

Por sua vez, o treinamento físico, também amplia a demanda metabólica, exerce efeito substancial sobre o balanço energético em seres humanos e em ratos ${ }^{14,15}$. Estudo com ratos submetidos a protocolo de treinamento físico com natação por 15 semanas mostrou menor peso corporal para o grupo treinado quando comparado com o grupo controle ${ }^{16}$. Ratos treinados com corrida em esteira por nove semanas, com intensidade ajustada para $65 \%$ do consumo máximo de oxigênio, mostraram redução estatística no peso corporal, comparado com o controle e, também, redução na quantidade ingerida de ração ${ }^{17}$.

Entretanto, poucos estudos foram realizados para avaliar a interação entre o fator exposição à fumaça de cigarro (EFC) e o fator treinamento físico, no apetite e no peso corporal de ratos. Desta forma, o objetivo do estudo foi de verificar a influência do tabagismo e do treinamento físico, no apetite e no desenvolvimento do peso corporal em ratos.

\section{MÉTODOS}

O protocolo experimental foi submetido e aprovado pelo pela Comissão de Ética no Uso de Animais (CEUA) da Universidade Federal do Espírito Santo (UFES) - protocolo no 062/2012. O protocolo de experimentação do estudo foi realizado na cidade de Vitória/ES, na Ufes, início em dezembro de 2013 (período experimental de 15 semanas).

Foram utilizados 40 ratos machos, da linhagem Wistar, pesando entre 200g e 250g, provenientes do Biotério do Centro de Ciências da Saúde da Universidade Federal do Espírito Santo. Todos os protocolos realizados seguiram o Guide for the Care and Use of Laboratory Animals publicado pelo U.S. National Institutes of Health.

Os animais foram distribuídos e randomizados em quatro grupos:

1) Grupo controle (C): Composto por dez 
animais, que não passaram por tratamento;

2) Grupo controle treinado (CT): Composto por dez animais, submetidos ao protocolo de treinamento físico por uma hora diária, durante cinco dias por semana. O exercício realizado foi à natação sem adição de sobrecarga de peso;

3) Grupo exposto (E): Composto por dez animais, os quais foram submetidos ao protocolo de exposição à fumaça de cigarro (EFC), sendo a taxa de 40 cigarros/dia.

4) Grupo treinado/exposto (TE): Composto por dez animais que passaram pelo protocolo de EFC sendo a taxa de 40 cigarros/dia. Submetido, também, ao protocolo de treinamento físico com duração de 1 hora/dia, durante cinco dias por semana. O exercício realizado foi à natação sem adição de sobrecarga de peso.

Após o processo de randomização, os animais foram alocados em cinco ratos por caixa. A temperatura ambiente foi controlada em torno de $22 \pm 2^{\circ} \mathrm{C}$ e ciclos de luminosidade de 12 horas claro/escuro, no período de 15 semanas.

Durante os primeiros 15 dias do experimento a oferta da ração (marca Labina ${ }^{\circledR}$ ) e água foi ad libitum para todos os grupos (C, CT, E e TE). A partir do $16^{\circ}$ dia a oferta da ração foi controlada, porém, a água continuou a ser ofertada ad libitum para todos os grupos. O grupo TE passou a ser referência para oferta de ração para o experimento, pois, observamos que sua ingestão de ração foi menor no período dos 15 dias iniciais. O grupo TE recebia diariamente $500 g$ de ração e, após a quantificação de seu consumo, a mesma quantidade de ração consumida pelo grupo referência era ofertada para os demais grupos (C, CT e E) do $16^{\circ}$ dia até o final do experimento (15 semanas).

\section{Peso Corporal}

Os animais foram pesados semanalmente, sempre no mesmo dia da semana, as segundasfeiras pela manhã, para acompanhamento da curva do desenvolvimento do peso corporal.

\section{Protocolo de Exposição à Fumaça de Cigarro}

O método utilizado de exposição crônica à fumaça de cigarro (EFC) foi descrito inicialmente por Simani ${ }^{10}$, adaptado por Paiva ${ }^{11}$ e utilizado por Castardeli ${ }^{12-14}$.

Os ratos foram colocados em câmaras transparentes, conectadas ao aparato de fumar com dimensão de aproximadamente $89 \times 86 \times 55 \mathrm{~cm}$. Os cigarros foram acesos e a fumaça aspirada por vácuo para a câmara onde estavam as caixas dos animais.

No período de adaptação, a fumaça de cigarro foi liberada a uma taxa de cinco cigarros por 30 minutos, durante os três primeiros dias, somente no período da manhã. No quarto, quinto e sexto dia de adaptação os animais foram expostos a uma taxa de cinco cigarros por 30 minutos uma vez no período da manhã e outra no período da tarde.

A partir do sétimo dia, o protocolo de EFC foi iniciado. O número de cigarros foi aumentado, assim como, a duração da EFC. Os animais passaram a serem expostos à fumaça a uma taxa de 10 cigarros por 30 min. Ao completar os 30 primeiros minutos de EFC foi dado intervalo de 10 minutos e retornando para a câmara de exposição para mais 30 minutos, repetindo a carga tabágica de 10 cigarros. O mesmo procedimento foi repetido no período da tarde, até o final do período de estudo.

O cigarro utilizado para exposição foi de marca comercial. Segundo o fabricante, os componentes básicos por cigarro utilizado são: mistura de fumos, açúcares, papel de cigarros, extratos vegetais, agentes de sabor, alcatrão $10 \mathrm{mg}$, nicotina - 0,8mg, monóxido de carbono $-10 \mathrm{mg}$.

\section{Protocolo de Treinamento}

O protocolo de treinamento físico utilizou a natação, o qual incluiu dois períodos, o de adaptação e o do treinamento propriamente dito. O período de adaptação durou dez dias e o de treinamento de três meses.

Na primeira semana de adaptação, os ratos foram submetidos à natação em água rasa e os animais apoiavam patas e cauda no fundo da piscina. Do primeiro ao sexto dia de adaptação os animais nadaram em água rasa com aumento progressivo do tempo, no primeiro e segundo dia os animais se exercitaram por dez minutos; terceiro e quarto dia, os ratos nadaram por 20 minutos; quinto e sexto dia os ratos nadaram 30 minutos. Na segunda semana de adaptação 
a altura da água foi o suficiente para evitar que os animais não encostassem as patas e/ ou a cauda no fundo da piscina. Sétimo dia de adaptação os animais nadaram 30 minutos, em água profunda. Oitavo dia os animais nadaram 40 minutos, nono dia 50 minutos e no décimo dia atingiram os 60 minutos de treinamento ${ }^{8}$.

Ao final do período de adaptação, iniciou-se o período de treinamento, que perdurou até o final do período experimental de três meses. As sessões eram na freqüência de cinco vezes por semana com durante 60 minutos por sessão diária.

A piscina de treinamento foi construída em vidro com 1,80 m de comprimento, 0,70m de altura e $0,60 \mathrm{~m}$ de largura dividida em seis raias, cada raia com $0,70 \mathrm{~m}$ de altura, 0,60 largura e 0,30 de comprimento.

Em cada raia foram colocados dois ratos, com temperatura da água variando de 30 a $34^{\circ} \mathrm{C}^{23}$.

Os animais do grupo TE tiveram intervalo mínimo de uma hora entre o protocolo de natação e a aplicação do protocolo de EFC, ou seja, ente aplicação do protocolo de natação para o de EFC respeitou-se um intervalo mínimo de uma hora, para que os ratos voltassem às condições de repouso.

\section{Análise Estatística}

Análise de variância (ANOVA) para o esquema de dois fatores, e complementado com o teste de comparação múltipla de Tukey. As análises foram efetuadas por meio de pacote estatístico SigmaStat, versão 3.5 (SPSS, San Raphel, CA, USA), admitindo-se nível de significância de 5\%.

\section{RESULTADOS}

Os resultados mostram menor ingestão de ração nos ratos EFC, tanto para o grupo $\mathrm{E}$ $(18,00 \mathrm{~g} \pm 0,7 \mathrm{~g})$ como para o grupo TE $(18,359 \mathrm{~g}$ $\pm 0,8 \mathrm{~g})$, com $p=0,001$, quando comparado com os grupos de ratos não exposto (grupos C - 23,50g \pm ,2g e CT - 21,367g $\pm 1,0 \mathrm{~g}$ ), durante 15 dias. Os ratos treinados (grupos CT e TE) ingeriram menores quantidades de ração quando comparados com os ratos não treinados (grupos C e E), com p=0,044. Ocorreu interação estatística entre os fatores (fumo e natação), para os mesmos 15 dias, com $p=0,013$ (figura 1-A).

Houve diferença na ingestão de ração ad libitum entre o grupo C comparado com o grupo CT $(p=0,002)$, do grupo C comparado com o grupo $E(p=0,001)$ e no grupo CT comparado com o grupo TE $(p=0,001)$. Somente não ocorreu diferença para a comparação entre $\mathrm{O}$ grupo TE comparado com o grupo $\mathrm{E}$, ambos os grupos expostos à fumaça de cigarro, com $\mathrm{p}=0,727$ (figura 1-B).

Para o peso inicial dos ratos não houve diferença entre os grupos estudados. Entretanto, os resultados apontaram diferenças estatísticas nas curvas do desenvolvimento do peso corporal entre os quatro grupos, no acompanhamento de 15 semanas, com valores inferiores de peso corporal nos grupos EFC (grupos E - 393,50g $\pm 12,46 \mathrm{~g}$ e TE - 393,33g $\pm 13,14 \mathrm{~g})$ e com valores superiores nos grupos não expostos grupo C $(451,00 \mathrm{~g} \pm 12,46 \mathrm{~g})$ e o grupo CT $(397,77 g \pm 13,14 \mathrm{~g})$. Houve interação estatística entre os fatores fumo e natação para o peso corporal dos ratos $(p=0,046)$ no acompanhamento de 15 semanas, conforme mostra a figura 2 .

Observaram-se menores valores para o peso do coração (VD $\pm \mathrm{VE})$, sem os átrios $(1,002 \mathrm{~g}$ $\pm 0,0308 \mathrm{~g})$ nos grupos expostos à fumaça de cigarro (grupo $\mathrm{E} \pm \mathrm{TE}$ ), em comparação com os ratos não expostos à fumaça de cigarro, os grupos $\mathrm{C}$, mais o grupo CT $(1,094 \mathrm{~g} \pm 0,0317 \mathrm{~g})$, figura 3.

No peso total do pulmão úmido (figura 4), observamos aumento significativo, para o fator exposição à fumaça de cigarro $(p=0,013)$ e, sucedeu também, diferença entre o grupo CT

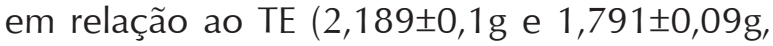
respectivamente, $p=0,008)$.

Ocorreu também diferença estatística no comprimento da tíbia (figura 5), para o fator exposição à fumaça de cigarro, bem como observamos os menores valores para o grupo $\mathrm{E}$ $(40,86 \pm 0,31 \mathrm{~mm})$ em relação ao grupo $C(42,47$ $\pm 0,33 \mathrm{~mm})$, com $p<0,001$. 


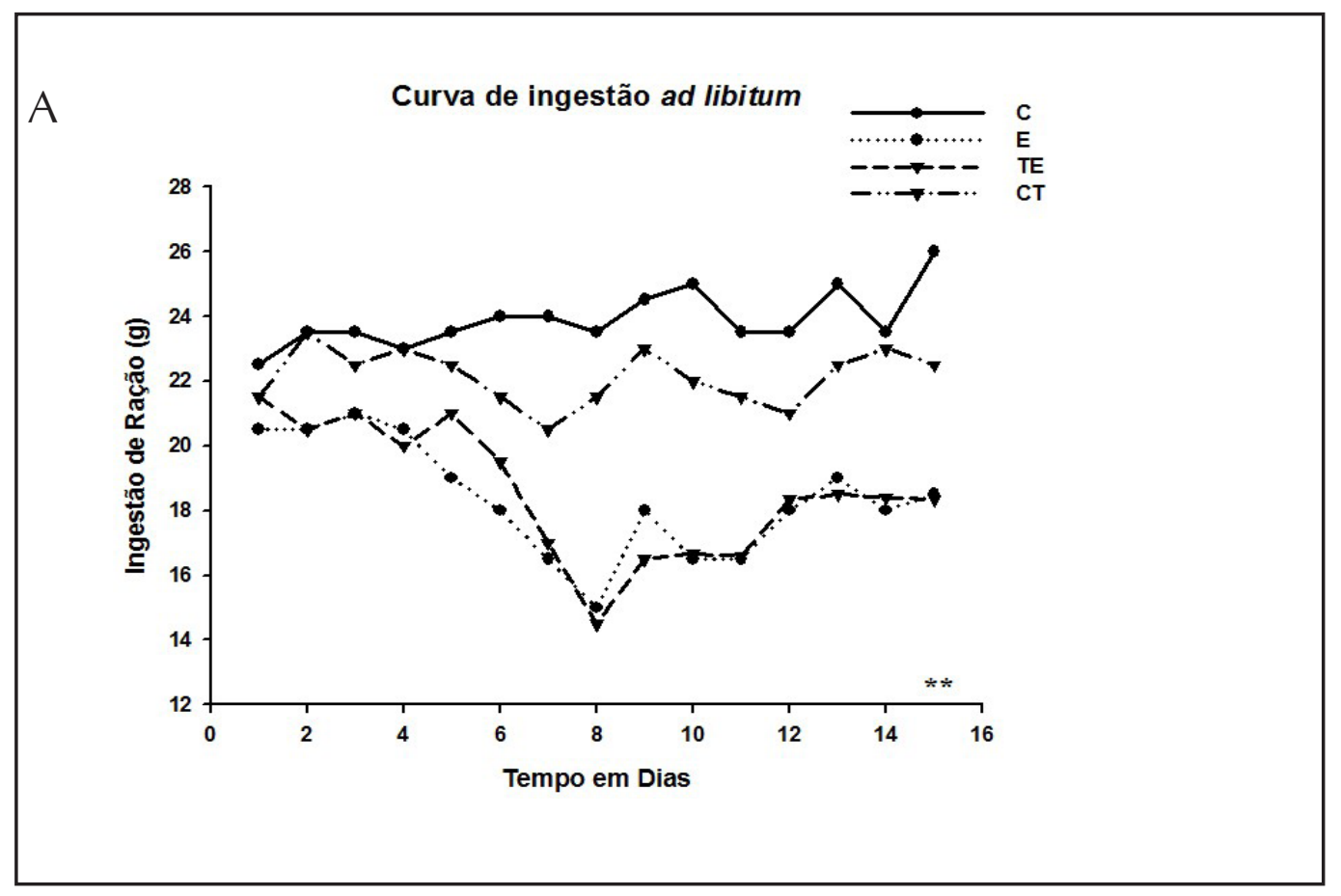

Figura 1 - A - Comparação das médias da ingestão de ração, entre os grupos expostos à fumaça de cigarro, os grupos treinados e a interação entre os dois fatores (treinado/exposto) - Vitória/ES, 11 junho de 2016.

${ }^{* *} p<0,05$ - para o fator exposição $(p=0,001)$; para o fator treinamento $(p=0,044)$; interação entre os fatores $(p=0,013)$.

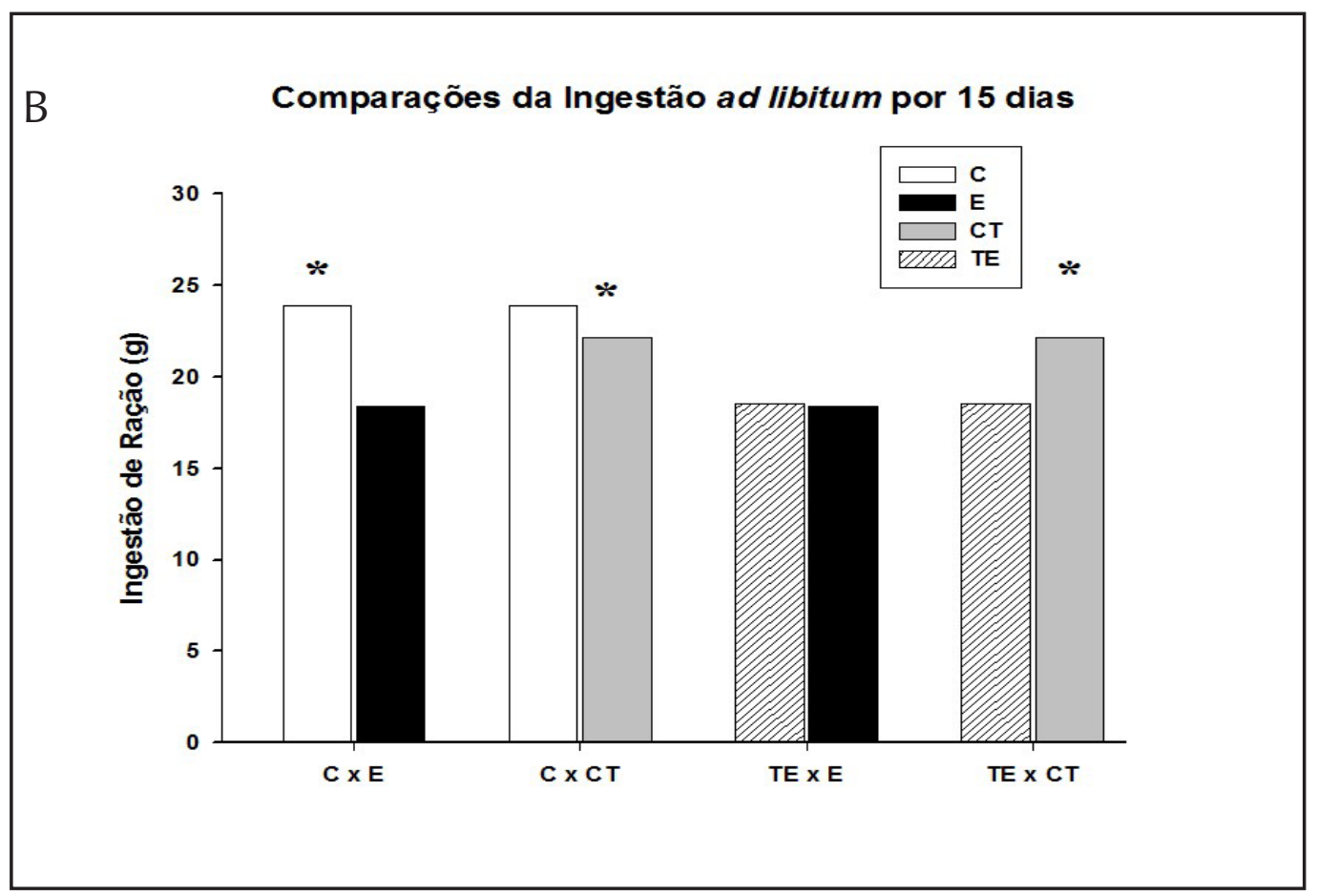

Figura 1 - B - Comparação das médias da ingestão de ração, entre os grupos experimentais. * $p<0,05$ 


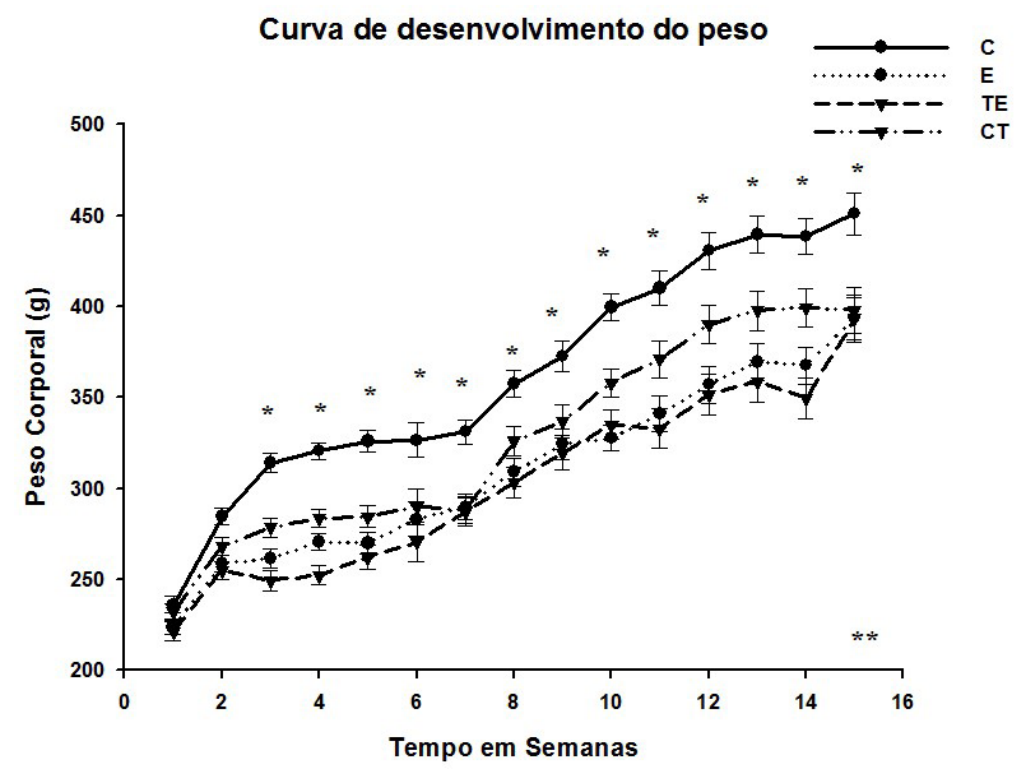

Figura 2 - Comparação das médias do peso corporal, entre os grupos expostos à fumaça de cigarro, os grupos treinados e a interação entre os dois fatores (treinado/exposto) - Vitória/ES, 11 junho de 2016. ** $p=0,009$ - para o fator exposição;

* $p<0,05$ - comparação do grupo controle com o grupo treinado.

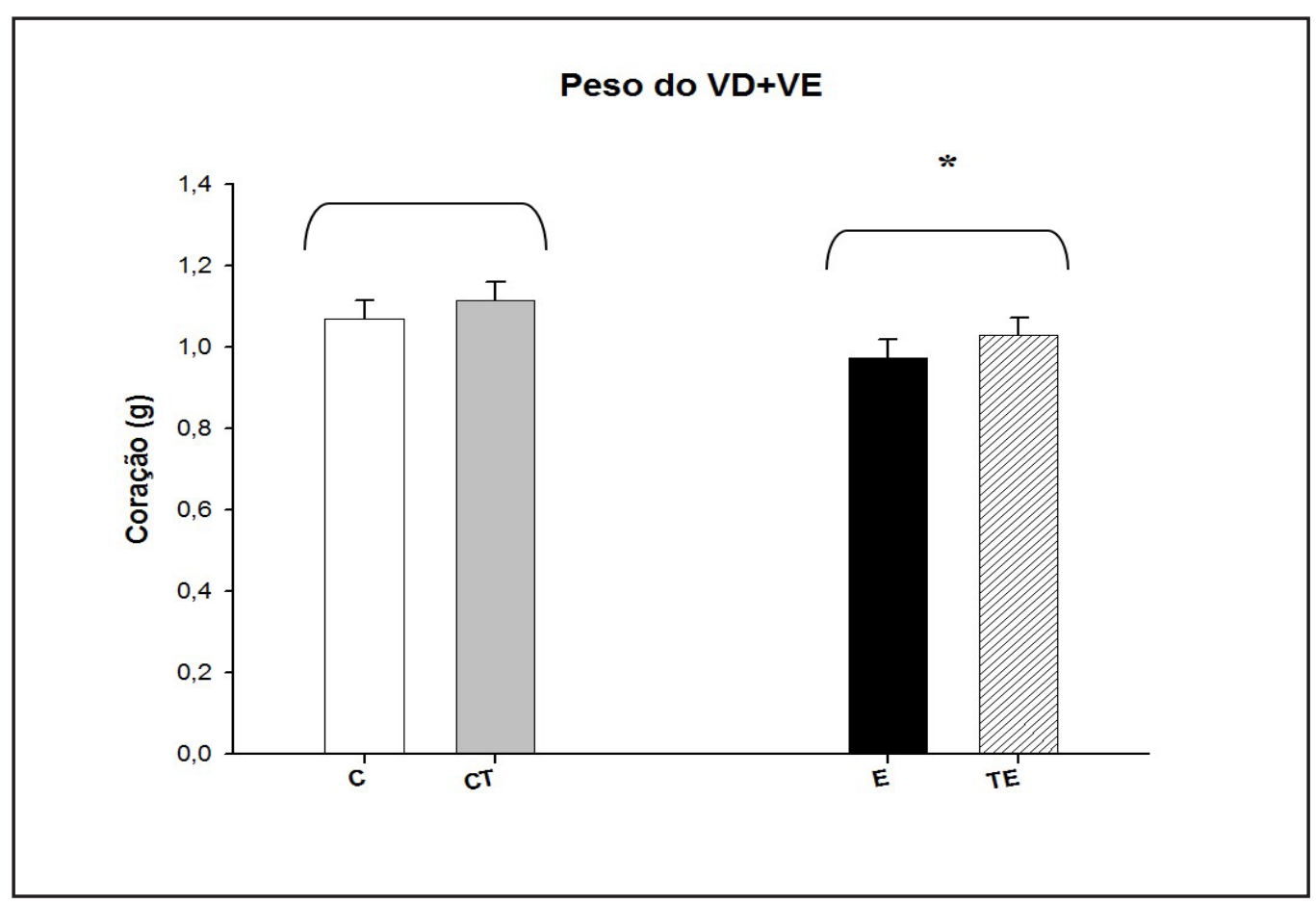

Figura 3 - Comparação das médias do peso do coração (VD+VE), entre os grupos não expostos à fumaça de cigarro (grupo $\mathrm{C}+$ grupo $\mathrm{CT}$ ) e os grupos exposto à fumaça de cigarro (grupo $\mathrm{E}+$ grupo TE) - Vitória/ES, 11 junho de 2016.

${ }^{*} p=0,047$ - para o fator exposição. 


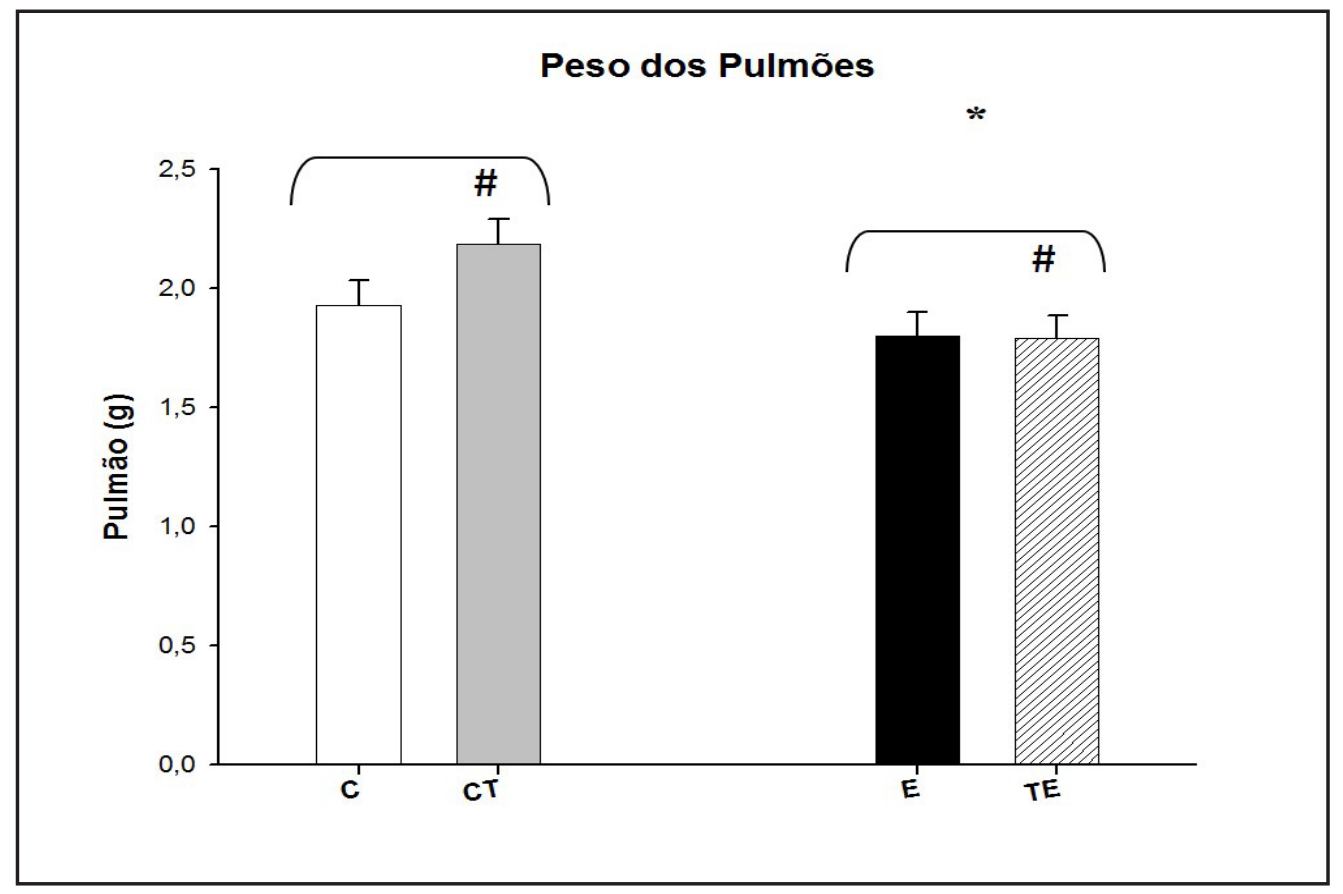

Figura 4 - Comparação das médias do peso do pulmão, entre os grupos não expostos à fumaça de cigarro (grupo $\mathrm{C}+$ grupo $\mathrm{CT}$ ) e os grupos exposto à fumaça de cigarro (grupo $\mathrm{E}+$ grupo TE), com * $p=0,013$ - para o fator exposição - Vitória/ES, 11 junho de 2016.

\# p=0,008 - para comparação entre o grupo Treinado e o Treinado/Exposto.

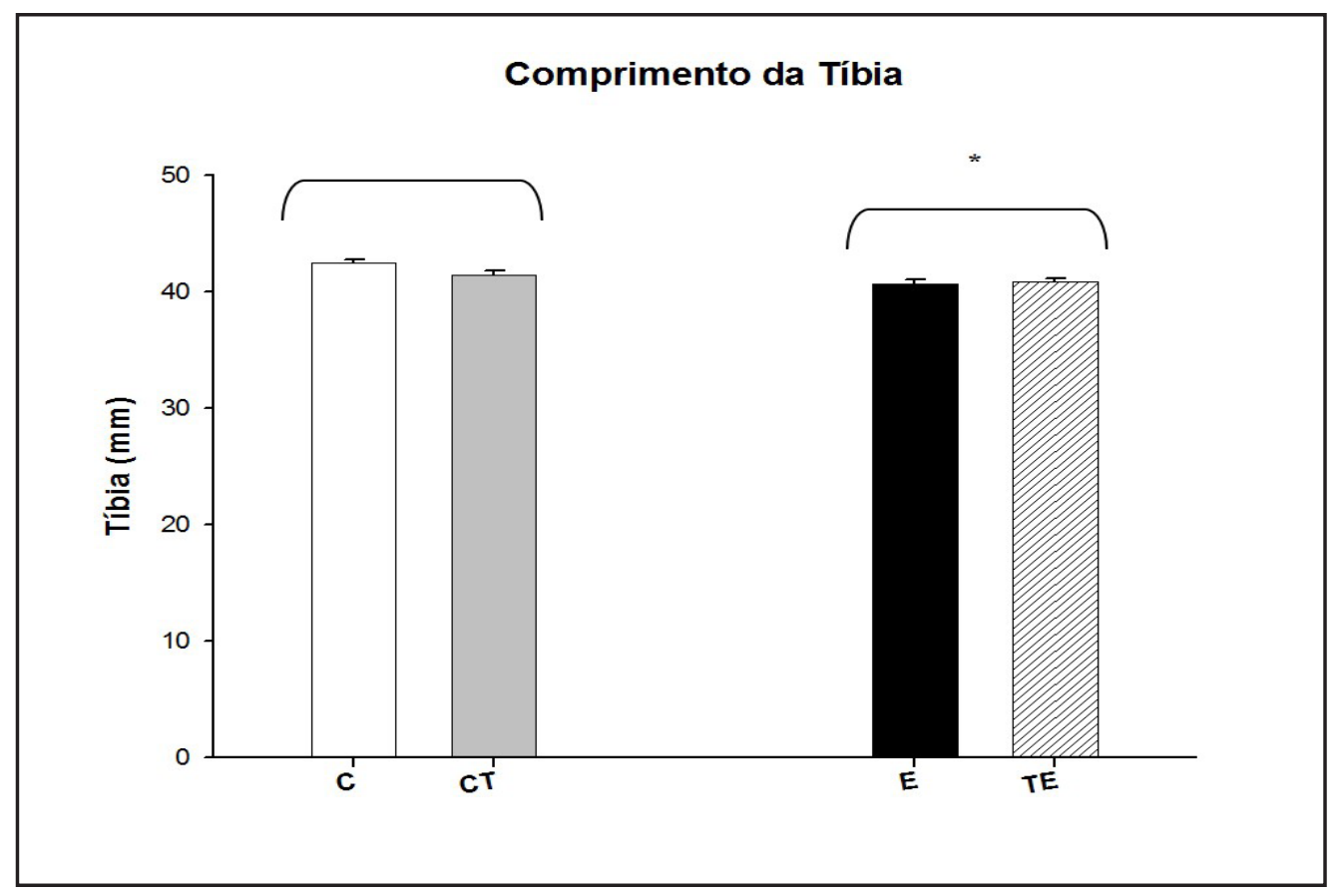

Figura 5 - Comparação das médias do comprimento da tíbia, entre os grupos não expostos à fumaça de cigarro (grupo $\mathrm{C}+$ grupo $\mathrm{CT}$ ) e os grupos exposto à fumaça de cigarro (grupo $\mathrm{E}+$ grupo TE) Vitória/ES, 11 junho de 2016.

* $p=0,001$ - para o fator exposição. 


\section{DISCUSSÃo}

Durante o período experimental, o peso dos animais foi monitorado semanalmente e a ração ingerida foi pesada diariamente. Dessa maneira, a análise da ingestão de ração ad libitum por 15 dias, evidenciou redução no apetite nos ratos expostos à fumaça de cigarro, de forma que, tanto os ratos do grupo E quanto do grupo TE obtiveram as menores médias de ingestão de ração durante 15 dias de observação, sem diferença estatística entre os respectivos grupos $(p=0,727)$.

Uma das possíveis explicações, para o ocorrido, é a influência exercida pelos inúmeros constituintes da fumaça de cigarro sobre o sistema hormonal, em especial, os controladores do apetite, pois, a nicotina induz o aumento da secreção de leptina pelo adipócito ${ }^{12,24,25}$.

Estudo em humanos fumantes ad libitum mostrou diminuição da concentração sérica de leptina, quando comparado com não fumantes. Entretanto, ao responderem a escala de Likert, que vai de um a sete ( $1=$ sem fome nem uma, $7=$ =xtrema fome), e determina o índice de fome, os maiores valores apareceram para os fumantes do grupo de abstinência por 24 horas e com diferença estatística quando comparado com os grupos não fumantes do estudo. Porém, a dieta desse estudo não foi controlada ${ }^{26}$.

Em estudo, com dieta não controlada apontou interferência na concentração sérica da leptina ${ }^{27}$. A dieta dos ratos de nosso estudo foi do mesmo fabricante, para todos os animais do estudo, por todo o período experimental.

Os mecanismos envolvidos no efeito da exposição à fumaça de cigarro no apetite e peso do corpo, não se restringe somente a uma única via. Um dos mecanismos candidatos da influência do fumo no apetite e peso corporal parece ser a interferência dos constituintes do fumo no sistema hormonal, em alguma de suas fases de produção ou sinalização (endócrina, autócrina e parácrina), devido à grande quantidade de constituintes presentes na fumaça de cigarro, isso, devido à quantidade excessiva de constituinte presente na fumaça de cigarro 4 .

Em nossos achados encontramos diferença entre as médias da ingestão de ração, para o fator treinamento $(p=0,044)$, com menores valores de ingestão para os grupos treinados e, também, diferença entre o grupo $\mathrm{C}$ em comparação ao grupo $\mathrm{CT}$, com p=0,002.

Os menores valores de ingestão apareceram no grupo $\mathrm{CT}$, esse achado foi surpreendente, pois esse grupo teve maior demanda calórica pelo fato de terem realizado o protocolo de natação. Entretanto, em estudo em humanos ao analisar uma única sessão de exercício em diferentes períodos do dia (pela manhã; à noite), com protocolo de treino com exercício aeróbio (20 min.) seguido de exercício condicionamento muscular (+20 min.), ao final da sessão de exercício os indivíduos acreditavam que "o exercício fez dar fome", pois os indivíduos relataram em escala visual de linha com 100 $\mathrm{mm}$, aumento da sensação de fome.

Portanto, nesse estudo esperou-se encontrar um aumento do apetite nos indivíduos póstreino e, consequentemente, aumento na ingestão calórica. Mas isso não ocorreu, pois, ao mensurar o consumo energético, no acompanhamento das refeições pós-treino, se verificou que não ocorreu aumento de consumo de energia. Dessa forma, o que aumentou de fato foi a sensação de fome ${ }^{28}$.

Uma possível explicação, para que não ocorra o aumento de ingestão calórica póstreino, seria o aumento da temperatura corpórea durante o período de treino e sua manutenção pós-treino. Assim, o aumento da sensação de fome não condiz com a real demanda calórica da necessidade fisiológica do organismo.

Em contrapartida, animais expostos ao frio apresentam demanda metabólica aumentada e com consequente aumento significativo de ingestão calórica, quando comparado com animais não expostos ao frio $^{29,30}$.

Ocorreu também, interação estatística entre os fatores fumo e treino $(p=0,013)$, ou seja, a soma dos dois estímulos modificou a média de ingestão de ração nos ratos que foram submetidos a exposição à fumaça de cigarro e ao protocolo de natação.

Dessa forma, o fumo se mostrou inibidor do apetite e o estímulo da natação não foi o suficiente para aumentar a ingestão de ração no grupo que recebeu o duplo estímulo (fumo e treino).

Ao analisar a curva de desenvolvimento do 
peso corporal dos animais, constatou-se que o fator exposição à fumaça de cigarro, associou-se a menos ganhos de peso corporal dos animais. Pois, houve redução do peso corporal dos grupos TE e F, ambos os grupos foram expostos à fumaça de cigarro (EFC).

Dados semelhantes foram encontrados em estudos realizados por Castardeli e colaboradores $^{20-21}$. Vale ressaltar que, o grupo TE foi o grupo referência para oferta de ração para todo experimento, ou seja, no grupo TE a ingestão de ração foi ad libitum. Os ratos do grupo $C$ receberam a mesma quantidade de ração que o grupo TE e, mesmo assim, o peso corporal dos ratos do grupo TE diminuiu significativamente, quando comparado com o grupo C. Esse fato indicou que a quantidade de ração ingerida foi o suficiente para garantir o desenvolvimento do peso corporal do grupo C e de todos os ratos dos grupos experimentais.

Uma das possíveis respostas para esse comportamento é a influência de nicotina, que é um dos principais constituintes viciantes presente na fumaça de cigarro $^{31}$. Além da nicotina influenciar o processo de dependência química, tem atuação sistêmica e altera o funcionamento do sistema nervoso central.

A nicotina aumenta a capacidade de consumo energético por meio da alteração do metabolismo basal e dos gastos com o exercício físico, além disso, induz o aumento da secreção de leptina pelo adipócito ${ }^{12,24,25}$.

Esta proteína está relacionada com a redução do apetite e com a regulação do peso corporal. Em estudo em animais com administração de nicotina houve aumento de $10 \%$ do dispêndio energético, com liberação de noradrenalina e serotonina que inibem o apetite.

A supressão nicotínica resulta na queda da taxa metabólica e estes fatos podem explicar, em parte, o aumento de peso nos ex-fumantes, e também o peso mais baixo nos fumantes ${ }^{32}$.

Em relação ao peso do grupo $\mathrm{CT}$, observamos que houve redução estatística e, para corroborar com nossos achados, o estudo realizado por lemitsu ${ }^{16}$, relataram que o treinamento físico de natação no período de 13 semanas influenciou na redução do peso dos animais treinados em relação aos grupos controle e ratos espontaneamente hipertensos (SHR).

O fator exposição à fumaça de cigarro influenciou no desenvolvimento de órgãos vitais, como o do coração (VD+VE) e no do pulmão. Assim como também, influenciou no comprimento da tíbia dos ratos EFC. Uma possível explicação do prejuízo no desenvolvimento do coração, pulmão e tíbia seria a aceleração do metabolismo corporal promovido pelo $\mathrm{EFC}^{12,24,25}$.

O metabolismo acelerado concorreu pela energia ingerida e, assim, ocorreu prejuízo no desenvolvimento corporal geral ${ }^{14,15}$.

O conjunto de nossos achados nos permite concluir que a exposição à fumaça de cigarro apresentou-se como inibidor do apetite e causou prejuízo no desenvolvimento corporal; o treinamento físico, na intensidade realizada interferiu na ingestão dos animais e no seu peso corporal; a interação entre os fatores fumo e treinamento influenciou no balanço energético negativo nos ratos estudados.

\section{CONCLUSÃO}

Conclui-se que apesar da exposição ao cigarro provocar diminuição da ingestão alimentar e diminuição de peso e, com isso, exercer aparente fator benéfico ao organismo, o fumo causou prejuízo no desenvolvimento físico dos ratos, com promoção de fator maléfico em órgãos vitais como coração e pulmões. Enquanto o treinamento físico provocou diminuição da ingestão e do peso corporal, sem alterar o desenvolvimento físico. Os benefícios advindos do treinamento físico não foram capazes de reverter os malefícios causados pelo fumo, pois a interação entre os fatores fumo e treinamento influenciou no balanço energético negativo nos ratos estudados, com consequente prejuízo no desenvolvimento corporal. 


\section{REFERÊNCIAS}

1- Dube M, Green CR. Methods of collection of smoke for analytical purpose. Recent Adv Tobacco Sci. 1982; 2: 42.

2- Green CR, Rodgmana. The tobacco chemist research for conference a half- century forum for advances inalytical methodology of tobacco and produs. Recent Adv Tobacco Sci. 1996; 22: 131.

3- Bonnie G. Coffa a, Christopher R.E. Coggins et al. Chemical, physical, and in vitro characterization of research cigarettes containing denicotinized tobacco. Regulatory Toxicology and Pharmacology 2016; 79: 64-73.

4- Smith CJ, Fisher TH. Particulate and vapor phase constituents of cigarette mainstream smoke and risk of myocardial infarction. Atherosclerosis 2001; 158: 257-67.

5- Stedman RL. The chemical composition of tobacco and tobacco smoke. Chem Rev. 1968; 153-207.

6- Friedman JM. The function of leptin in nutrition, weight, and physiology. Nutr Rev. 2002; 60: 1-14.

7- Nicklas BJ, Tomoyasu N, Muir J, Goldberg AP. Effects of cigarette smoking and its cessation on body weight and plasma leptin levels. Metabolism. 1999; 48(6): 804-8.

8- Perkins KA, Fonte C. Effects of smoking status and smoking cessation on leptin levels. Nicotine Tob Res. 2002; 4(4): 459-66. 9- Eliasson B, Smith U. Leptin levels in smokers and long-term users of nicotine gum. Eur J Clin Invest. 1999; 29(2): 145-52

10- Albanes D, Jones DY, Micozzi MS, Mattson ME. Associations between smoking and body weight in the US population: analysis of NHANES II. Am J Public Health. 1987;77(4):439-44.

11- Klesges RC, Ward KD, Ray JW, Cutter G, Jacobs DR Jr, Wagenknecht LE. The prospective relationships between smoking and weight in a young, biracial cohort: the Coronary Artery Risk Development in Young Adults Study. J Consult Clin Psychol. 1998; 66(6): 987-93.

12- Cunha, G. H.; Jorge, A. R. C.; Fonteles, M. M. F.; Sousa, F. C. F.; Viana, G. S. B. \& Vasconcelos, S. M. M. Nicotina e tabagismo. Revista Eletrônica Pesquisa Médica 2007, 1 (4).

13- Dórea AJP, Botelho C. Fatores dificultadores de cessação do tabagismo. J BrasPneumol 2004. Aug: 30(2): 41-6.

14- Whybrow S, Hughes DA, Ritz P, Johnstone AM, Horgan GW, King N, Blundell JE, et al. The effect of an incremental increase in exercise on appetite, eating behaviour and energy balance in lean men and women feeding ad libitum. $\mathrm{Br} J$ Nutr. 2008; 100: 1109-15. DOI: 10.1017/S0007114508968240. <http://journals.cambridge.org/action/ displayAbstract?fromPage $=$ online\&aid $=2411284 \&$ fileld $=$ S0007114508968240>

15- Bergouignan A, Momken I, Schoeller DA, Normand S, Zahariev A, Lescure B, et al. Regulation of energy balance during long-term physical inactivity induced by bed rest with and without exercise training. J Clin Endocrinol Metab. 2010; 95: 1045-53. DOI: 10.1210/ jc.2009-1005.

16- lemitsu M, Miyauchi T, Maeda S, Sakai S, Kobayashi T, Fujii N, et al. Physiological and pathological cardiac hypertrophy induce different molecular phenotypes in rats. Am J Physiol Regulatory Integrative Comp Physiol. 2001; 281: 2029-36.

17- Zhao J, Tian Y, Xu J, Liu D, Wang X, Zhao B. Endurance exercise is a leptin signaling mimetic in hypothalamus of Wistar rats. Lipids in Health and Disease 2011, 10: 225. DOI: 10.1186/1476-511X-10-225. < http://lipidworld.biomedcentral.com/articles/10.1186/1476511X-10-225>

18- Simani AS, Inoue S, Hoggs JC. Penetration of the respiratory epithelium of guinea pigs following exposure to cigarette smoke. Lab Invest. 1974; 31: 75-81.

19- Paiva SAR, Zornoff LAM, Okoshi MP, Okoshi K, Cicogna AC, Campana AO. Comportamento de variáveis cardíacas em animais expostos à fumaça de cigarro. Arq Bras Cardiol. 2003; 81: 221-4.

20- Castardeli E, Paiva SA, Matsubara BB, Matsubara LS, Minicucci MF, Azevedo OS, et al. Chronic cigarette smoke exposure results in cardiac remodeling and impaired ventricular function in rats. Arq. Bras Cardiol. 2005; 84(4): 320-4.

21- Castardeli E, Duarte RD, Minicucci FM, Azevedo SP, Matsubara BB, Matsubara SL, Álvaro O, et al. Tobacco-smoke-induced left ventricular remodeling is not associated with metalloproteinase-2 or -9 activation. Eur J Heart Failure. 2007; 9: 1081-5. DOI: 10.1016/j. ejheart.2007.09.004.

22- Castardeli, E, Duarte DR, Minicucci MF, Azevedo SP, Matsubara BB, Matsubara SL, Álvaro O, et al. Exposure time and ventricular remodeling induced by tobacco smoke exposure in rats. Med Sci Monit. 2008; 14(3): 62-6.

23- Portes LA, Tucci PJF. O treinamento físico por natação atenua o remodelamento miocárdico e congestão pulmonar em ratas wistar com insuficiência cardíaca secundária a infarto do miocárdio. Arq. Bras Cardiol. 2005; 87(1): 57-8.

24- Haxby, DG, Rodriguez, GS, Zechnich AD, Schuff RA, Tanigawa JS. Treatment of nicotine dependence. Am J Health Syst Pharm. 1995; v52 (3): 265-81.

25- Marques ACPR, Campana A, De Paula Gigliotti A, Lourenço MTC, Ferreira MP, Laranjeira R. Consensus on the treatment of nicotine dependence. Rev Bras Psiquiatr. 2001; 23(4): 200-14.

26- Klein LC, Corwin EJ, Ceballos RM. Leptin, hunger, and body weight: Influence of gender, tobacco smoking, and smoking abstinence. Addict Behav. 2004; 29: 921-7. DOI: 10.1016/j.addbeh.2004.02.023. <http://www.sciencedirect.com/science/article/pii/ S0306460304000309>

27- Oeser, A, Goffaux J, Snead W, Carlson MG. Plasma leptin concentrations and lipid profiles during nicotine abstinence. Am J Med Sci. 1999; 318(3): 152-7.

28- Maraki M, Tsofliou F, Pitsiladis YP, Malkova D, Mutrie N, Higgins S. Acute effects of a single exercise class on appetite, energy intake and mood. Is there a time of day effect? Appetite 2005; 45: 272-8. DOI: 10.1016/j.appet.2005.07.005. <http://www.sciencedirect. com/science/article/pii/S0195666305000991>

29- Hori S, Hori K, Kaya M, Ishigaki T, Koyama K, Otani H, et al. Comparison of body mass, food intake and plasma constituents in lean and obese Zucker rats under cold acclimation and deacclimation. J Therm Biol. 2007; 32(6): 363-4. DOI: 10.1016/j. jtherbio.2007.05.003. <http://eurekamag.com/research/004/409/004409316.php>

30- Zhang XY, Jing BB, Wang DH. Cold exposure does not decrease serum leptin concentration, but increases energy intake and thermogenic capacity in pregnant Brandt's voles (Lasiopodomys brandtii). Zoology 2009; 112(3): 206-16. DOI: 10.1016/j. zool.2008.09.003. <http://www.sciencedirect.com/science/article/pii/S0944200609000038>

31- US Department of Health and Human Services. The health consequences of smoking: nicotine addiction: A report from the Surgeon General. Washington DC: Government Printing Office; 1988 [DHHS Publication CDC 88-8406]. 
32- Nerín I, Beamonte A, Gargallo P, Jiménez-Muro A, Marqueta A. Weight Gain and Anxiety Levels in Recent Ex-Smokers. Arch Bronconeumol. 2007; 43: 01. DOI: 10.1157/13096995. <http://www.sciencedirect.com/science/article/pii/S1579212907600147 > 